The Astrophysical Journal, 652:120-125, 2006 November 20

(C) 2006. The American Astronomical Society. All rights reserved. Printed in U.S.A.

\title{
CONTRIBUTION OF STELLAR TIDAL DISRUPTIONS TO THE X-RAY LUMINOSITY FUNCTION OF ACTIVE GALAXIES
}

\author{
Miloš Milosavljević, ${ }^{1,2}$ David Merritt, ${ }^{3}$ and Luis C. $\mathrm{Ho}^{4}$ \\ Received 2006 February 16; accepted 2006 August 3
}

\begin{abstract}
The luminosity function of active galactic nuclei (AGNs) has been measured down to luminosities $\sim 10^{42} \mathrm{ergs} \mathrm{s}^{-1}$ in the soft and hard X-rays. Some fraction of this activity is associated with the accretion of the material liberated by the tidal disruption of stars by massive black holes. We estimate the contribution to the X-ray luminosity function from the tidal disruption process. While the contribution depends on a number of poorly known parameters, it appears that it can account for the majority of X-ray-selected AGNs with soft or hard X-ray luminosities $\lesssim 10^{43}-10^{44} \mathrm{ergs} \mathrm{s}^{-1}$. If this is correct, a significant portion of the X-ray luminosity function of AGNs is comprised of sources powered by tidal disruption at the faint end, while the sources at the bright end are powered by nonstellar accretion. Black holes with masses $\lesssim 2 \times 10^{6} M_{\odot}$ could have acquired most of their present mass by an accretion of tidal debris. In view of the considerable theoretical uncertainty concerning the detailed shape of the light curves of tidal disruption events, we focus on power-law luminosity decay (as identified in candidate tidal disruption events), but we also discuss constant accretion rate models. We expect that the sources associated with thin-disk accretion of circularized tidal debris have peak luminosities of $\leqslant 10^{39}-10^{41} \mathrm{ergs} \mathrm{s}^{-1}$, below the luminosity range probed in present surveys.
\end{abstract}

Subject headings: accretion, accretion disks — black hole physics — cosmology: observations galaxies: active — galaxies: nuclei

\section{INTRODUCTION}

A main-sequence or giant star passing near a massive black hole $(\mathrm{MBH})$ of mass $M_{\mathrm{bh}} \lesssim 10^{8} M_{\odot}$ is disrupted by the tidal forces (Hills 1975). The disruption and the subsequent accretion of stellar debris by the black hole are potentially powerful sources of X-ray emission (e.g., Mészáros \& Silk 1977; Young et al. 1977; Rees 1988, 1990). The emission can be produced in various phases of tidal disruption, including the initial compression of the star (Kobayashi et al. 2004), the interaction of ejected debris with the ambient medium (Khokhlov \& Melia 1996), tidal stream collisions and debris fallback onto a nascent accretion disk (Kochanek 1994; Lee et al. 1996; Kim et al. 1999; Li et al. 2002), and the accretion of disk material onto the black hole (Cannizzo et al. 1990, hereafter CLG90; Ulmer 1999). The purpose of the present study is to estimate the contribution of activity associated with tidal disruptions to the luminosities of typical active galaxies.

Recently, surveys with the High Energy Astronomical Observatory (HEAO-1), Advanced Satellite for Cosmology and Astrophysics (ASCA), Chandra, and XMM-Newton have yielded luminosity functions (LFs) of active galaxies at low and high redshift in the hard X-rays (e.g., Ueda et al. 2003; Barger et al. 2005) and soft X-rays (e.g., Hasinger et al. 2005). The measured $\mathrm{X}$-ray LFs extend to lower luminosities than their optical counterparts because contamination with host galaxy light makes optical detection of low-luminosity AGNs difficult. The LFs are approximately broken power laws $d N / d \ln L_{\mathrm{X}} \propto L_{\mathrm{X}}^{\mu}\left(L_{\mathrm{X}}+L_{\mathrm{X} \star}\right)^{\nu-\mu}$ with $\nu \approx-2$ and $-1 \lesssim \mu \lesssim 0$, and $L_{\mathrm{X} \star} \sim 10^{43}-10^{44} \mathrm{ergs} \mathrm{s}^{-1}$. The power-law behavior at the bright end has been attributed to the

\footnotetext{
1 Department of Theoretical Astrophysics, California Institute of Technology, Mail Code 130-33, 1200 East California Boulevard, Pasadena, CA 91125. 2 Hubble Fellow.

3 Department of Physics, Rochester Institute of Technology, 84 Lomb Memorial Drive, Rochester, NY 14623.

4 Observatories of the Carnegie Institution of Washington, 813 Santa Barbara Street, Pasadena, CA 91101.
}

intrinsic structure of quasar luminosity histories. The luminosity histories (light curves) are convolved with the black hole mass function to calculate the LF (e.g., Wyithe \& Loeb 2003; Hopkins et al. 2005). The origin of the LF at the faint end, however, remains unknown.

At least a fraction of low-luminosity AGNs must be powered by stellar tidal disruption. Here we present a first estimate of the contribution of these sources to the X-ray LF of AGNs. This estimate is an essential step to understanding the origin of activity in AGNs with black hole masses $\lesssim 10^{7} M_{\odot}$ and ultimately to understanding the origin of low-mass MBHs.

Theoretical investigations of the stellar tidal disruption process, and the detection of X-ray flares that are candidate tidal disruption events (e.g., Komossa \& Greiner 1999; Komossa et al. 2004; Halpern et al. 2004), suggest that the nuclei in which stellar tidal disruption has occurred remain luminous for $1-10 \mathrm{yr}$ after disruption or longer. The integrated luminosity of the AGN depends on the mass of the stellar debris that remains bound to the black hole, and on the fraction of this mass that ends up accreting onto the black hole in a radiatively efficient fashion. The bound mass can be a fraction $f \sim 0.25-0.5$ of the initial stellar mass (e.g., Ayal et al. 2000).

The bound mass inferred in the giant X-ray flare in NGC 5905 using a blackbody fallback model is much smaller than the solar mass ( $\mathrm{Li}$ et al. 2002); in two other candidate flares, RX J1624.9+ 7554 and RX J1242.6-1119A, the light curves are consistent with masses $\sim 1 M_{\odot}$. Donley et al. (2002) constrained the rate of outbursts by comparing the Röntgensatellit (ROSAT) All-Sky Survey with archival pointed observations. They inferred a rate of $\sim 10^{-5} \mathrm{yr}^{-1}$ per galaxy. We adopt a theoretical estimate for the tidal disruption rate that is significantly higher (eq. [1]).

In principle, the signatures of X-ray activity associated with tidal disruption events may not be restricted to the duration of the X-ray flare. After most of the bound tidal debris has been accreted and the continuum ionizing source has waned, the transient activity may still be detected in the ionization state of the interstellar medium. Eracleous et al. (1995) pointed out that the decay 
timescales for certain low-ionization states can be $\sim 100 \mathrm{yr}$ and suggested that the transient ionizing activity associated with tidal disruptions may lead to low-ionization nuclear emissionline regions (LINERs). Pogge et al. (2000) tested this scenario with spatially resolved narrowband imaging of 14 LINERs with the Hubble Space Telescope (HST). The imaging has produced no evidence for the expected annular geometry of $\mathrm{O}$ III emission predicted in the transient excitation model.

The outline of our calculation of the AGN LF is as follows. In $\S 2.1$ we estimate the mass function of MBHs and the rate of tidal disruptions. In $\S 2.2$ we present simple models for the light curves of tidally disrupted stars. In $\S 2.3$ we calculate the LF and explore its dependence on various parameters in the model. And in $\S 3$ we discuss implications of our estimate for the understanding of low-luminosity, X-ray-selected AGNs.

\section{CALCULATION OF THE LUMINOSITY FUNCTION}

\subsection{Tidal Disruption Rate}

Stars passing within the tidal distance $r_{t} \sim\left(\eta^{2} M_{\mathrm{bh}} / m_{\star}\right)^{1 / 3} R_{\star}$ from the black hole, where $m_{\star}$ is the mass of the star, $R_{\star}$ is its radius, and $\eta \sim 1$ is a numerical factor, are tidally disrupted. Here we estimate the tidal disruption rate in a galaxy with black hole of mass $M_{\mathrm{bh}}$ and stellar velocity dispersion $\sigma$. The disruption rate depends on the detailed structure of the stellar nucleus of the galaxy. The galaxies relevant to this study are those with $M_{\mathrm{bh}} \lesssim$ $M_{\text {max }} \equiv 10^{8} M_{\odot}\left(R_{\star} / R_{\odot}\right)^{3 / 2}\left(m_{\star} / M_{\odot}\right)^{-1 / 2}$, which can disrupt stars.

In spheroids as faint as that of the Milky Way, the relaxation time within the black hole's radius of dynamical influence $r_{\mathrm{bh}} \sim$ $G M_{\mathrm{bh}} / \sigma^{2}$ is short enough that a collisionally relaxed distribution, $\rho \propto r^{-\gamma}$, where $\gamma \sim 1.5-1.75$, is set up (Bahcall \& Wolf 1976, 1977; Merritt \& Szell 2006). This is consistent with what is seen at the Galactic center (Genzel et al. 2003; Schödel et al. 2006). But just beyond $r_{\text {bh }}$, the Galactic center density profile steepens to $\rho \propto r^{-2}$, and this is also the slope observed with the HST near the centers of all but the most luminous galaxies (Gebhardt et al. 1996; Ferrarese et al. 2006). Furthermore, nuclear relaxation times in galaxies with $M_{\mathrm{bh}} \gtrsim 10^{7} M_{\odot}$ generally exceed a Hubble time (Faber et al. 1997); thus, a Bahcall-Wolf cusp would not form.

The rate of stellar disruptions in stellar density cusps has been studied at various levels of detail (e.g., Frank \& Rees 1976; Lightman \& Shapiro 1977; Cohn \& Kulsrud 1978; Magorrian \& Tremaine 1999). We adopt the most recent estimate of the rate in a $\rho \propto r^{-2}$ cusp (Wang \& Merritt 2004),

$$
\begin{aligned}
\Gamma\left(M_{\mathrm{bh}}\right) \approx & \times 10^{-4} \mathrm{yr}^{-1}\left(\frac{\sigma}{70 \mathrm{~km} \mathrm{~s}^{-1}}\right)^{7 / 2}\left(\frac{M_{\mathrm{bh}}}{10^{6} M_{\odot}}\right)^{-1} \\
& \times\left(\frac{m_{\star}}{M_{\odot}}\right)^{-1 / 3}\left(\frac{R_{\star}}{R_{\odot}}\right)^{1 / 4},
\end{aligned}
$$

where $m_{\star}$ and $R_{\star}$ are the mass and radius of the tidally disrupted stars.

The disruption rate in equation (1) must be weighted by the stellar mass function in the spheroid at distances from the black hole corresponding to the initial pericenter radii of tidally disrupted stars. These radii are typically similar to $r_{\text {bh }}$ (Magorrian \& Tremaine 1999). If the stellar mass function resembles the "universal" initial mass function (Kroupa 2001), the disruption is dominated by $m_{\star} \sim 0.1 M_{\odot}$ stars. However, the mass function near the black hole may differ from the universal initial mass function because higher mass stars dynamically segregate closer to a $\mathrm{MBH}$, and because stars with an unusual initial mass function may form in situ near the MBH (e.g., Milosavljević \& Loeb 2004 and references therein).

Mounting observational evidence indicates that the black hole mass and the velocity dispersion of the host stellar spheroid are tightly correlated with the best-fit relation $M_{\mathrm{bh}} \approx 1.7 \times$ $10^{8} M_{\odot}\left(\sigma / 200 \mathrm{~km} \mathrm{~s}^{-1}\right)^{4.9}$ (e.g., Ferrarese \& Ford 2005 and references therein). We explore departures from this fiducial relation by parameterizing the logarithmic slope of the relation $M_{\mathrm{bh}} \propto \sigma^{a}$.

No direct constraints on the cosmic density of a $\mathrm{MBH}$ with masses $\lesssim 10^{7} M_{\odot}$ exist. The majority of these MBHs should be in spiral galaxies. The density can be estimated by assuming a relation between the bulge luminosity and the black hole mass of the form $L_{\text {bulge }}=A M_{\mathrm{bh}}^{k}$. Following Ferrarese (2002) we substitute this in the Schechter LF, $\Phi(L) d L=\Phi_{0}\left(L / L_{\star}\right)^{\alpha} e^{-L / L_{\star}} d L / L_{\star}$, in order to obtain a cosmic mass function of black holes,

$$
\Psi\left(M_{\mathrm{bh}}\right) d M_{\mathrm{bh}}=\Psi_{0}\left(\frac{M_{\mathrm{bh}}}{M_{\star}}\right)^{k(\alpha+1)-1} e^{-\left(M_{\mathrm{bh}} / M_{\star}\right)^{k}} \frac{d M_{\mathrm{bh}}}{M_{\star}}
$$

where $\Psi_{0}=k \Phi_{0}, M_{\star}=\left(1.27 \beta L_{\star} / A\right)^{1 / k}$, and $\beta \equiv L_{\text {bulge }} / L \sim 0.3$ for the Hubble type Sab (Simien \& de Vaucouleurs 1986), which we use as reference. The latter is justified because spiral galaxies dominate galaxy LF below $\sim L_{\star}$ (e.g., Nakamura et al. 2003). For the relation between the blue luminosity of the galaxy and the black hole mass, we adopt $L=3900 L_{\odot}\left(M_{\mathrm{bh}} / M_{\odot}\right)^{0.79}$ (Marconi \& Hunt 2003).

The number density of $\sim 10^{8} M_{\odot}$ black holes estimated using equation (2) agrees with a similar estimate in Marconi et al. (2004), while the number density of $\sim 10^{6} M_{\odot}$ black holes exceeds their estimate by a factor $\sim 3-4$. This may be a consequence of our using the $B$-band galaxy LF as reference, whereas Marconi et al. (2004) used the $K$-band LF that is flatter at the faint end. We adopt a steeper $M_{\mathrm{bh}}-\sigma$ relation $(a=4.9)$ than they did $(a=4.11)$. Since the $M_{\mathrm{bh}}-\sigma$ relation is normalized at $M_{\mathrm{bh}} \sim 10^{8} M_{\odot}$, a larger $a$ results in a higher velocity dispersion at fixed black hole mass $M_{\mathrm{bh}}<10^{8} M_{\odot}$, which in turn implies a higher tidal disruption rate in equation (1). This underscores some uncertainties inherent in an attempt to extrapolate the statistics of $\mathrm{MBH}$ populations to low masses. The validity of the extrapolation is particularly imperiled by the assumption that relations such as $M_{\mathrm{bh}}-L$ are pure power laws, whereas significant departures from the power-law behavior are of course inevitable.

\subsection{The Light Curve of a Tidal Disruption Event}

The light curves of candidate tidal disruption events (Komossa 2004 and references therein) are characterized by rapid fall in $\mathrm{X}$-ray luminosity on timescales of months to a year, followed by a gradual continued fading over the following decade. Three of the four candidate events (NGC 5905, RX J1420+53, RX J1242-11) are consistent with luminosity decay $L_{\mathrm{fb}} \propto t^{-5 / 3}$ expected if the $\mathrm{X}$-ray emission is produced during the fallback of stellar debris onto a nascent accretion disk (Phinney 1989; Evans \& Kochanek 1989). The fourth event, RX J1624+75, exhibits what appears to be faster initial decay followed by very slow fading. In all cases, the total drop in X-ray luminosity is by a factor $\sim 10^{2}-10^{3}$ over about a decade. Gezari et al. (2003) did follow-up optical spectroscopy of RX J1420+53 and RX J1242-11 with HST and found no evidence for emission lines or a nonstellar continuum emission. In NGC 5905, however, they found nuclear emission lines that suggest a persistent, albeit particularly variable Seyfert nucleus, which possibly undermines the case for the tidal disruption interpretation in NGC 5905. 
The above candidate tidal disruption events have been selected for characteristic, "outburst-like" light curves (fast rise and slow decay). However, the true luminosity evolution of tidal disruption events is unknown and may be different from that of the candidate events. If the emission during the fallback stage is absorbed or otherwise suppressed, the X-ray light curve will be dominated by thin-disk accretion, and a quasi-steady flux over a longer period will be expected. Such events would remain undetected in existing searches that target outburst activity. To maintain generality, therefore, we consider fallback-like power-law light curves and also discuss constant accretion rate models.

The power irradiated during fallback was derived in $\mathrm{Li}$ et al. (2002),

$L_{\mathrm{bol}} \approx 4 \times 10^{42} \operatorname{ergs~s}^{-1}\left(\frac{f}{0.25}\right)\left(\frac{M_{\mathrm{bh}}}{10^{6} M_{\odot}}\right)\left(\frac{m_{\star}}{M_{\odot}}\right)^{2 / 3}\left(\frac{t}{1 \mathrm{yr}}\right)^{-5 / 3}$,

where the tidal disruption occurs at $t=0$, and the luminosity reaches peak value and starts decaying as in equation (3) at time

$$
t_{\text {peak }} \approx 0.02 \mathrm{yr}\left(\frac{M_{\mathrm{bh}}}{10^{6} M_{\odot}}\right)^{1 / 2}\left(\frac{m_{\star}}{M_{\odot}}\right)^{-1}\left(\frac{R_{\star}}{R_{\odot}}\right)^{3 / 2}
$$

after tidal disruption.

Although equation (3) was derived for the fallback process, we interpret it as a representative power-law decay model for the luminosity evolution, independent of the emission mechanism. To test the sensitivity to the power-law index of luminosity decay, we consider decay of the form $L_{\mathrm{bol}} \propto t^{-\lambda}$, where $\lambda>1$ is a constant. The time between tidal disruption and peak luminosity in equation (4) was derived under the assumption that the tidal stream collides with itself at the first pericenter passage. The selfcollision may be avoided temporarily at the first passage because of the relativistic Lense-Thirring precession (Kochanek 1994). Therefore, we also test the sensitivity to the value of $t_{\text {peak }}$ by scaling it via $t_{\text {peak }}(\xi)=\xi t_{\text {peak }}(0)$. In both cases, we keep the total energy irradiated between $t=t_{\text {peak }}$ and $t=\infty$ constant and equal to $f \epsilon m_{\star} c^{2}$, where $\epsilon$ is a radiative efficiency factor.

As in Li et al. (2002), the radiative efficiency is calculated by assuming that the fallback material releases its kinetic energy promptly at the circularization radius that coincides with the tidal disruption radius, $\epsilon=\left(G m_{\star} / 4 R_{\star} c^{2}\right)\left(M_{\mathrm{bh}} / m_{\star}\right)^{2 / 3} \ll 1$. The ultimate accretion of the circularized material onto the black hole can of course produce much higher efficiency, $\epsilon \sim 0.1-0.4$, depending on the accretion rate, on the black hole spin, and on the orientation of the accretion disk relative to the black hole spin. The higher efficiency, however, does not imply higher luminosity in thin-disk-powered sources, as we detail below.

We also discuss an alternative to power-law light curves. Accurate theoretical modeling of a time-dependent thin-disk emission is difficult. We therefore adopt a crude quasi-steady accretion scenario, in which we assume that accretion at a constant rate proceeds "while the supplies last," i.e., until all the bound debris has been accreted. In this scenario, we assume that the luminosity is a fraction $\ell$ of the Eddington luminosity $L_{\mathrm{Edd}}\left(M_{\mathrm{bh}}\right)$ and that the luminosity is related to the mass accretion rate via $L=\epsilon \dot{M} c^{2}$, where as usual $\epsilon$ parameterizes radiative efficiency. The duration of the active phase is then $\Delta t_{\text {active }}=\epsilon f m_{\star} c^{2} / \ell L_{\text {Edd }}$, implying a model luminosity $L_{\text {bol }}\left(M_{\text {bh }}, t\right)=\ell L_{\text {Edd }} \mathrm{H}\left(\Delta t_{\text {active }}-t\right)$, where $\mathrm{H}(x)$ is the Heaviside step function.

Constant accretion rate is inevitably an oversimplification. Taking into account the redistribution of mass in the disk, CLG90 estimated that under certain assumptions the light curve will rise gradually on a viscous timescale of the accretion disk and then decay as a power law, $L_{\text {disk }} \propto t^{-1.2}$. Menou \& Quataert (2001) pointed out, however, that if outer parts of the disk become thermally unstable and neutral, the accretion will be delayed. This caveat aside, the value of the peak bolometric luminosity that we can infer in Figure 3 of CLG90 is approximately given by

$$
L_{\mathrm{CLG} 90, \text { peak }} \sim\left(5 \times 10^{43}\right) \alpha_{\text {disk }}\left(\frac{f m_{\star}}{0.5 M_{\odot}}\right) \mathrm{ergs} \mathrm{s}^{-1},
$$

where $\alpha_{\text {disk }}$ is the stress-to-pressure ratio in the disk, and a black hole $M_{\mathrm{bh}}=10^{7} M_{\odot}$ was assumed. Magnetohydrodynamic simulations of turbulent radiation-pressure-supported accretion disks suggest $\alpha_{\text {disk }} \sim 0.01-0.001$ (e.g., Turner 2004), which implies a very small peak luminosity $L_{\text {peak }} \leqslant 5 \times 10^{41} \mathrm{ergs} \mathrm{s}^{-1}$. CLG90 did not explore the dependence of $L_{\text {peak }}$ on the mass of the black hole, but we expect smaller black holes to yield higher peak luminosities (at a fixed $f m_{\star}$ ) because the star is disrupted at a smaller radius, and the resulting accretion disk is hotter. This, however, is probably insufficient to raise the peak bolometric luminosity into the range $\left(L \gtrsim 10^{43} \mathrm{ergs} \mathrm{s}^{-1}\right)$ relevant for comparison with the X-ray luminosity function data.

\subsection{Integration over the Black Hole Mass Function}

The LF of tidal disruption events is obtained by weighting the disruption probability modeled as a Poisson process by the black hole mass function. In the fallback model, the LF reads

$$
\begin{aligned}
\Psi\left(L_{\mathrm{X}}\right)= & \int_{M_{\min }}^{M_{\max }\left(m_{\star}\right)} d M_{\mathrm{bh}} \int_{t_{\mathrm{paak}}\left(M_{\mathrm{bh}}\right)}^{\infty} d t \Psi\left(M_{\mathrm{bh}}\right) \Gamma\left(M_{\mathrm{bh}}\right) e^{-\Gamma\left(M_{\mathrm{bh}}\right) t} \\
& \times \delta\left[\omega^{-1} L_{\mathrm{bol}}\left(M_{\mathrm{bh}}, t\right)-L_{\mathrm{X}}\right]
\end{aligned}
$$

where $\omega$ is the bolometric correction, $\delta(x)$ is the Dirac $\delta$-function, and the dependence on $f$ and $m_{\star}$ is implicitly assumed; for the radius of subsolar stars, we adopt the relation $R_{\star}=R_{\odot}\left(m_{\star} / M_{\odot}\right)^{0.8}$ (e.g., Kippenhahn \& Weigert 1990). Here, $M_{\min }$ is the minimum MBH mass, and as before $M_{\max } \propto m_{\star}^{0.7}$ is the maximum MBH mass near which a star can be disrupted. We take $M_{\min }=10^{4} M_{\odot}$, as systems, in which an even smaller black hole is expected, have been found to lack a black hole (e.g., Merritt et al. 2001; Valluri et al. 2005). For the bolometric correction in the $0.5-2 \mathrm{keV}$ band, we take $\omega=45$, which at low luminosities is approximately independent of luminosity (Marconi et al. 2004 and references therein), although in reality the bolometric correction could be correlated with $L_{\mathrm{bol}} / L_{\mathrm{Edd}}$ rather than $L_{\mathrm{bol}}$.

In Figure 1, we plot the resulting LF for our fiducial model with a light curve that decays as a power law $L \propto t^{-\lambda}$ with $\lambda=\frac{5}{3}$. The model is based on a galaxy LF with amplitude $\Phi_{0}=$ $0.02^{3} h^{-3} \mathrm{Mpc}^{-3}$ and absolute blue magnitude of an $L_{\star}$ galaxy of $M_{B \star}=-20.0+5 \log _{10} h$ (Blanton et al. 2001), where we assume $h=0.7$. We vary the various parameters, including $\lambda$ in Figure $1 d$, to explore the sensitivity of the X-ray AGN LF to our choice of the fiducial model. The set of the parameters employed in this paper is summarized in Table 1. In the low-luminosity regime $L_{\mathrm{X}} \ll L_{\text {peak }} \equiv \omega^{-1} L_{\text {bol }}\left[m_{\star}, t_{\text {peak }}\left(m_{\star}\right)\right]$, the LF is a power law, $\Psi\left(L_{\mathrm{X}}\right) \propto L_{\mathrm{X}}^{-1 / \lambda-1}$. At $L_{\mathrm{X}} \gtrsim L_{\text {peak }} \sim$ $10^{44}(\omega / 45)^{-1}\left(m_{\star} / M_{\odot}\right)^{1 / 3}$ ergs s $^{-1}$, the LF drops precipitously.

Below $L_{\mathrm{X}} \sim 10^{44} \mathrm{ergs} \mathrm{s}^{-1}$, the calculated X-ray AGN LF slightly overestimates but is roughly compatible with the soft X-ray AGN LF of Hasinger et al. (2005; shown in Fig. 1), and is a factor of a few lower than the hard X-ray AGN LF (Ueda et al. 2003; Barger et al. 2005). The discrepancy between the soft and 


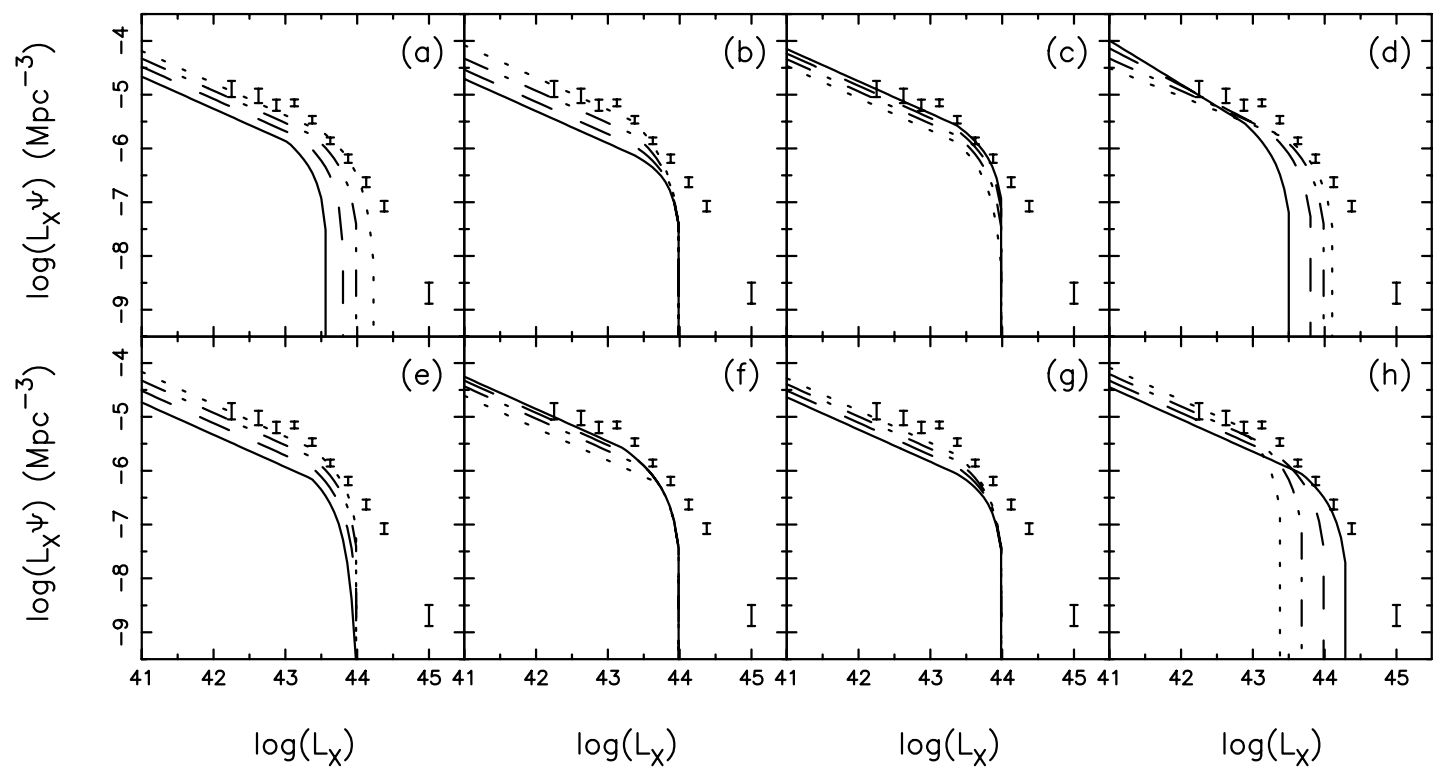

FIG. 1.-X-ray LF of tidal disruptions calculated using eq. (6), assuming a bolometric correction $\omega=45$ and a light curve that decays as a power law, $\lambda=\frac{5}{3}$. In each panel, a parameter is varied to illustrate dependence: $(a) m_{\star}=(0.1,0.333,1.0,3.0) M_{\odot} ;(b) \alpha=(-1,-1.15,-1.3,-1.45) ;(c) k=(0.70,0.75,0.8,0.85) ;(d) \lambda=$ $(1.222,1.444,1.666,1.888) ;(e) M_{B \star}=(-18,-19,-20,-21)+5 \log _{10} h ;(f) M_{\min }=\left(10^{3}, 10^{4}, 10^{5}, 10^{6}\right) M_{\odot} ;(g) a=(4.0,4.333,4.666,5.0) ;$ and $(h) \xi=(0.5$, $1,2,4)$, where the fiducial value has been italicized and the four parameter values correspond to the solid, the dashed, the dot-dashed, and the dotted lines, respectively. We also show the low-redshift $(0.015<z<0.2)$ soft X-ray $(0.5-2 \mathrm{keV})$ LF data from the Chandra and XMM-Newton survey of Hasinger et al. (2005). A Hubble constant of $H_{0}=70 \mathrm{~km} \mathrm{~s}^{-1} \mathrm{Mpc}^{-1}$ is assumed throughout.

hard X-ray LF could be attributed to an incompleteness of the soft X-ray LF resulting from obscuration.

In models with power-law-decaying light curves, the lowluminosity slope of the AGN LF is determined by the power law of the decay, rather than the shape of the black hole mass function (Fig. 1d). The amplitude of the LF, however, is sensitive to the black hole mass function. The steep drop in the LF around $10^{44} \mathrm{ergs} \mathrm{s}^{-1}$ is associated with the peak luminosity produced in a tidal disruption event, which corresponds to the peak mass inflow rate from the debris that is beginning to circularize around the black hole (Figs. $1 a$ and $1 h$ ).

In the "while the supplies last" model, where the accretion luminosity is a constant and equal to $\ell L_{\mathrm{Edd}}$, the $\mathrm{LF}$ reads

$$
\Psi\left(L_{\mathrm{X}}\right) \approx \frac{\epsilon f}{\ell} \frac{\sigma_{\mathrm{T}} c}{4 \pi G m_{p}} \frac{m_{\star}}{L_{\mathrm{X}}} \Gamma\left(M_{1}\right) \Psi\left(M_{1}\right),
$$

for $M_{\min }<M_{1}<M_{\max }$, where $M_{1}=\omega \sigma_{\mathrm{T}} L_{\mathrm{X}} / 4 \pi \ell G m_{p} c$. In the low-luminosity regime, the LF is a power law, $\Psi\left(L_{\mathrm{X}}\right) \propto$ $L_{\mathrm{X}}^{-3+7 / 2 a+(\alpha+1) k} \ell^{1-7 / 2 a-(\alpha+1) k}$, which, assuming a constant $\ell$, is steeper $\left[L_{\mathrm{X}} \Psi\left(L_{\mathrm{X}}\right) \propto L_{\mathrm{X}}^{-1.5}\right]$ than the observed relation $(\mu>-1)$. However, the two LFs have comparable amplitudes at $L_{\mathrm{X}} \sim$ $10^{43} \mathrm{ergs} \mathrm{s}^{-1}$. The discrepancy may reflect a luminosity-dependent Eddington ratio $\ell$. The dependence of $\ell$ on luminosity or black hole mass is expected if the accretion rate is supply-limited, rather than radiatively limited. For example, to reproduce $\Psi\left(L_{\mathrm{X}}\right) \propto L_{\mathrm{X}}^{-2}$, we must have $\ell \propto L_{\mathrm{X}}$. At present, it is not clear how such dependence should arise.

The low efficiency of accretion through a thin $\alpha$-disk, discussed in $\S 2.2$, implies that the maximum luminosity expected from disk emission is also very low. This puts into question the ability of the late, postcircularization accretion of tidal debris to contribute significantly to the low-luminosity part of the measured

TABLE 1

Parameters Entering the Calculation of $\Psi\left(L_{\mathrm{X}}\right)$

\begin{tabular}{|c|c|c|}
\hline Parameter & Symbol & Fiducial Value \\
\hline Stellar mass $\left(M_{\odot}\right) \ldots \ldots$ & $m_{\star}$ & 1 \\
\hline$\left\{r_{t} /\left[\left(M_{\mathrm{bh}} / m_{\star}\right)^{1 / 3} R_{\star}\right]\right\}^{3 / 2}$. & $\eta$ & 1 \\
\hline Fraction of tidal debris bound to $\mathrm{MBH}$ & $f$ & 0.25 \\
\hline 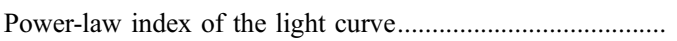 & $\lambda$ & $5 / 3$ \\
\hline$t_{\text {peak }}$ in units of that in eq. (4) & $\xi$ & 1 \\
\hline 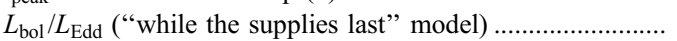 & $\ell$ & 1 \\
\hline 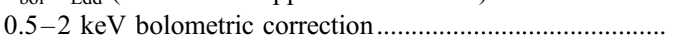 & $\omega$ & 45 \\
\hline Normalization of galaxy LF $\left(h^{-3} \mathrm{Mpc}^{-3}\right) \ldots \ldots \ldots \ldots \ldots \ldots \ldots \ldots \ldots$ & $\Phi_{0}$ & 0.02 \\
\hline Low-luminosity power-law index of galaxy LF ...................... & $\alpha$ & -1.3 \\
\hline Absolute $B$ magnitude of galaxy LF turnoff............................. & $M_{B \star}$ & $-20+5 \log _{10}$ \\
\hline 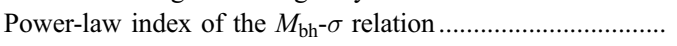 & $a$ & 4.9 \\
\hline 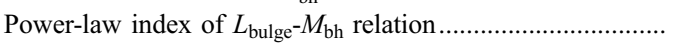 & $k$ & 0.8 \\
\hline 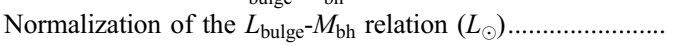 & $A$ & 3900 \\
\hline 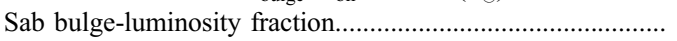 & $\beta$ & 0.3 \\
\hline Minimum black hole mass $\left(M_{\odot}\right)$ & $M_{\mathrm{bh}}$ & $10^{4}$ \\
\hline
\end{tabular}


X-ray LF. The thin-disk phase is unavoidable in the accretion of the debris, but the associated sources should appear at luminosities below the current lower completeness limit of the surveys at $L_{\mathrm{X}, \min } \sim 10^{42} \mathrm{ergs} \mathrm{s}^{-1}$. We do not attempt to calculate the luminosity function of thin-disk-powered sources.

\section{DISCUSSION}

While many theoretical uncertainties frustrate an accurate estimate of the contribution of activity associated with stellar tidal disruption to the X-ray LF of active galaxies, it seems that the contribution can be significant at low luminosities, $L_{X} \lesssim$ $10^{44} \mathrm{ergs} \mathrm{s}^{-1}$. The tidal disruption activity makes a negligible contribution at higher luminosities (power-law profile of the measured soft X-ray LF extends to $L_{\mathrm{X}} \sim 10^{47.5} \mathrm{ergs} \mathrm{s}^{-1}$ at redshifts $z \sim 1-3$ ). MBHs with masses above $\sim 10^{7} M_{\odot}$ grow by accreting nonstellar material from the interstellar medium.

What is the origin of the featureless knee in the LF at $L_{\mathrm{X}} \sim$ $\left(10^{43}-10^{44}\right) \mathrm{ergs}^{-1}$ ? The location of the knee coincides with Eddington-limited accretion onto black holes with masses $M_{\mathrm{bh}} \sim$ $10^{6}-10^{7} M_{\odot}{ }^{5}$ Given the tidal disruption rate assumed here (eq. [1]), black holes with masses $M_{\mathrm{bh}} \lesssim 2 \times 10^{6}(f / 0.25) M_{\odot}$, where $f$ is the fraction of the stellar mass that ultimately gets accreted onto the $\mathrm{MBH}$, could have grown to their present size by accreting stellar debris over a Hubble time, although undoubtedly nonstellar accretion must have played a role. This critical mass is compatible with the location of the knee in the LF, assuming near-Eddington accretion.

Black holes with masses $\sim 10^{7} M_{\odot}$, in turn, roughly lie at the demarcation line separating the dominance of late-type and earlytype galaxies. If major mergers are responsible for diverting gas into accretion onto black holes in early-type galaxies, then the absence of merging activity in late-type galaxies leaves stellar tidal disruption as a competitive supplier of material to the $\mathrm{MBH}$. This may explain why the steep power-law behavior of the quasar LF fails to extend to lower luminosities.

The observed X-ray LF of AGNs evolves with redshift. The number density of low-luminosity AGNs increases by a factor of $\sim 10$ at $L_{\mathrm{X}} \sim 10^{42} \mathrm{ergs} \mathrm{s}^{-1}$ and a factor of $\sim 4$ at $L_{\mathrm{X}} \sim$ $10^{43} \mathrm{ergs} \mathrm{s}^{-1}$ from $z \sim 0$ to $z \sim 0.7$ in the soft X-rays $(0.5-$ $2 \mathrm{keV}$; Hasinger et al. 2005), while the same increase is only a factor $\sim 2-3$ in the hard X-rays (2-8 keV; Barger et al. 2005). The location of the knee shifts from $L_{\mathrm{X}} \sim 10^{43} \mathrm{ergs} \mathrm{s}^{-1}$ to $L_{\mathrm{X}} \sim$ $10^{44} \operatorname{ergs~s}^{-1}$ from $z \sim 0$ to $z \sim 1$ in the soft and hard band alike, and the break becomes more prominent at higher redshift. These trends require explanation.

The number density of tidal-disruption-powered sources could increase with redshift because of one or more of the following reasons:

1. On average, MBHs are smaller at larger redshift, implying a larger tidal disruption rate or, speculatively, a higher radiative efficiency in the soft X-ray band. ${ }^{6}$

\footnotetext{
5 The maximum accretion rate might exceed the Eddington limit by a factor of a few (e.g., Begelman 2002).

${ }^{6}$ Note that for black holes smaller than $M_{\mathrm{bh}} \lesssim 10^{6} M_{\odot}$, thermal emission from an Eddington-limited disk enters the soft X-ray band, and the soft X-ray luminosity is thus anticorrelated with mass. It is also possible that transition from high- to low-state accretion is sensitive to $M_{\mathrm{bh}}$.
}

2. Galactic nuclei were denser at larger redshift (or, equivalently, stellar velocity dispersions were higher at fixed $\mathrm{MBH}$ mass), also implying a larger tidal disruption rate.

3. Tidally disrupted stars were more massive at larger redshift, implying brighter events. ${ }^{7}$

Any one or all of these factors may contribute to the observed trend at low luminosities, roughly below the knee of the LF at $L_{\mathrm{X}} \sim 10^{43.5} \mathrm{ergs} \mathrm{s}^{-1}$. Kinematical limitations on the mass inflow rate imply that luminosities above the knee are not produced during the circularization and accretion of debris from disrupted solar-mass stars. The maximum luminosity generated in a tidal disruption event corresponds to the rate at which the most closely bound debris loses orbital energy to circularize around the black hole. ${ }^{8}$ This luminosity is coincidentally comparable to the luminosity of the knee of the X-ray LF.

If MBHs grew gradually in preexisting stellar density cusps with fixed velocity dispersions, then the tidal disruption rate would be inversely proportional to the black hole mass (eq. [1]) and would increase with redshift. This could explain the increase in the number density of low-luminosity AGNs from the nearby universe to $z \sim 0.7$.

The relative contribution of stellar tidal disruptions to the X-ray AGN LF can be studied by examining long-term X-ray variability of the sources. Currently, long-term monitoring data have been analyzed for only a handful of sources (e.g., Markowitz \& Edelson 2004). Theoretical uncertainty notwithstanding, the light curves of tidal disruption events should be characterized by a fast rise and slow decay, but the variability may or may not be detected as an outburst. The frequency of hard X-ray outbursts in the ROSAT All-Sky Survey was studied by Donley et al. (2002). Their estimate, $\sim 10^{-5} \mathrm{yr}^{-1}$ per galaxy, is significantly below the tidal disruption rate adopted by us. The rates can be reconciled if the majority of tidal disruptions do not exhibit outburst characteristics. In that case, a measurable asymmetry in the autocorrelation function would still be expected.

We would like to thank an anonymous referee for helpful comments and Avishay Gal-Yam, Eran Ofek, Sterl Phinney, and Ohad Shemmer for inspiring discussions. M. M. acknowledges support by NASA through the Hubble Fellowship grant HSTHF-01188.01-A awarded by the Space Telescope Science Institute, which is operated by the Association of Universities for Research in Astronomy, Inc., for NASA under contract NAS526555. D. M. acknowledges support by NSF grants AST 0420920 and AST 04-37519, and NASA grant NNG04GJ48G.

\footnotetext{
7 The remaining possibility, an increase of the number of black holes in the centers of galaxies with redshift, seems implausible.

${ }^{8}$ Kobayashi et al. (2004) estimated that the peak X-ray luminosity resulting from the dissipation of the tidal-compression-induced shock in the surface layers of the star is $\lesssim 10^{42}\left(m_{\star} / M_{\odot}\right)^{19 / 12}\left(R_{\star} / R_{\odot}\right)^{-5 / 4}\left(M_{\mathrm{bh}} / 10^{6} M_{\odot}\right)^{1 / 6} \mathrm{ergs} \mathrm{s}^{-1}$; but the duration of this event is brief (measured in seconds) and is not detectable in conventional surveys.
}

Ayal, S., Livio, M., \& Piran, T. 2000, ApJ, 545, 772

Bahcall, J. N., \& Wolf, R. A. 1976, ApJ, 209, 214 1977, ApJ, 216, 883

Barger, A. J., Cowie, L. L., Mushotzky, R. F., Yang, Y., Wang, W.-H., Steffen, A. T., \& Capak, P. 2005, AJ, 129, 578

\section{REFERENCES}

Begelman, M. C. 2002, ApJ, 568, L97

Blanton, M. R., et al. 2001, AJ, 121, 2358

Cannizzo, J. K., Lee, H. M., \& Goodman, J. 1990, ApJ, 351, 38 (CLG90)

Cohn, H., \& Kulsrud, R. M. 1978, ApJ, 226, 1087

Donley, J. L., Brandt, W. N., Eracleous, M., \& Boller, T. 2002, AJ, 124, 1308 
Eracleous, M., Livio, M., \& Binette, L. 1995, ApJ, 445, L1

Evans, C. R., \& Kochanek, C. S. 1989, ApJ, 346, L13

Faber, S. M., et al. 1997, AJ, 114, 1771

Ferrarese, L. 2002, in Current High-energy Emission around Black Holes, ed.

C.-H. Lee \& H.-Y. Chang (Singapore: World Scientific), 3

Ferrarese, L., \& Ford, H. 2005, Space Sci. Rev., 116, 523

Ferrarese, L., et al. 2006, ApJ, 644, L21

Frank, J., \& Rees, M. J. 1976, MNRAS, 176, 633

Gebhardt, K., et al. 1996, AJ, 112, 105

Genzel, R., et al. 2003, ApJ, 594, 812

Gezari, S., Halpern, J. P., Komossa, S., Grupe, D., \& Leighly, K. M. 2003, ApJ, 592,42

Halpern, J. P., Gezari, S., \& Komossa, S. 2004, ApJ, 604, 572

Hasinger, G., Miyaji, T., \& Schmidt, M. 2005, A\&A, 441, 417

Hills, J. G. 1975, Nature, 254, 295

Hopkins, P. F., Hernquist, L., Cox, T. J., Di Matteo, T., Robertson, B., \& Springel, V. 2005, ApJ, 630, 716

Kim, S. S., Park, M.-G., \& Lee, H. M. 1999, ApJ, 519, 647

Kippenhahn, R., \& Weigert, A. 1990, Stellar Structure and Evolution (Berlin: Springer)

Kobayashi, S., Laguna, P., Phinney, E. S., \& Mészáros, P. 2004, ApJ, 615, 855

Kochanek, C. S. 1994, ApJ, 422, 508

Khokhlov, A., \& Melia, F. 1996, ApJ, 457, L61

Komossa, S. 2004, in IAU Symp. 222, The Interplay among Black Holes, Stars and ISM in Galactic Nuclei, ed. T. S. Bergmann, L. C. Ho, \& H. R. Schmitt (Cambridge: Cambridge Univ. Press), 45

Komossa, S., \& Greiner, J. 1999, A\&A, 349, L45

Komossa, S., Halpern, J., Schartel, N., Hasinger, G., Santos-Lleo, M., \& Predehl, P. 2004, ApJ, 603, L17

Kroupa, P. 2001, MNRAS, 322, 231
Lee, H. M., Kang, H., \& Ryu, D. 1996, ApJ, 464, 131

Li, L.-X., Narayan, R., \& Menou, K. 2002, ApJ, 576, 753

Lightman, A. P., \& Shapiro, S. L. 1977, ApJ, 211, 244

Magorrian, J., \& Tremaine, S. 1999, MNRAS, 309, 447

Marconi, A., \& Hunt, L. K. 2003, ApJ, 589, L21

Marconi, A., Risaliti, G., Gilli, R., Hunt, L. K., Maiolino, R., \& Salvati, M. 2004, MNRAS, 351, 169

Markowitz, A., \& Edelson, R. 2004, ApJ, 617, 939

Menou, K., \& Quataert, E. 2001, ApJ, 562, L137

Merritt, D., Ferrarese, L., \& Joseph, C. L. 2001, Science, 293, 1116

Merritt, D., \& Szell, A. 2006, ApJ, 648, 890

Mészáros, P., \& Silk, J. 1977, A\&A, 55, 289

Milosavljević, M., \& Loeb, A. 2004, ApJ, 604, L45

Nakamura, O., Fukugita, M., Yasuda, N., Loveday, J., Brinkmann, J., Schneider, D. P., Shimasaku, K., \& SubbaRao, M. 2003, AJ, 125, 1682

Phinney, E. S. 1989, in IAU Symp. 136, The Center of the Galaxy, ed. M. Morris (Dordrecht: Kluwer), 543

Pogge, R. W., Maoz, D., Ho, L. C., \& Eracleous, M. 2000, ApJ, 532, 323

Rees, M. J. 1988, Nature, 333, 523 1990, Science, 247, 817

Schödel, R., Eckart, A., Genzel, R., Merritt, D., Alexander, T., Ott, T., \& Straubmeier, C. 2006, A\&A, submitted

Simien, F., \& de Vaucouleurs, G. 1986, ApJ, 302, 564

Turner, N. J. 2004, ApJ, 605, L45

Ueda, Y., Akiyama, M., Ohta, K., \& Miyaji, T. 2003, ApJ, 598, 886

Ulmer, A. 1999, ApJ, 514, 180

Valluri, M., Ferrarese, L., Merritt, D., \& Joseph, C. L. 2005, ApJ, 628, 137

Wang, J., \& Merritt, D. 2004, ApJ, 600, 149

Wyithe, J. S. B., \& Loeb, A. 2003, ApJ, 595, 614

Young, P. J., Shields, G. A., \& Wheeler, J. C. 1977, ApJ, 212, 367 